\title{
Vận dụng sự phân rã DuPont vào chỉ số ROA: Bằng chứng thực nghiệm về rủi ro hoạt động của các ngân hàng thương mại tại Việt Nam Application of DuPont decomposition to risks of ROA Index: Empirical evidence of Banks in Vietnam
}

\author{
Nguyễn Thành Hưng ${ }^{1 *}$ \\ ${ }^{1}$ Công ty TNHH Vật liệu xây dựng Vạn Phát, Việt Nam \\ "Tác giả liên hệ, Email: thanhhung.tg@gmail.com
}

THÔNG TIN

DOI: $10.46223 /$ HCMCOUJS. econ.vi.15.1.254.2020

Ngày nhận: 07/10/2019

Ngày nhận lại: 12/11/2019

Duyệt đăng: 15/11/2019

Tù khóa:

Dupont decomposition, OLS, ROA

Keywords:

Dupont decomposition, OLS, ROA

\section{TÓM TẮT}

Mục tiêu của bài viết vừa là nghiên cứu tìm ra sự giới hạn của chỉ số ROA. Dựa trên cách tiếp cận mô hình DuPont, các yếu tố cấu thành trong công thức phản ánh sự đóng góp của yếu tố đầu vào cho yếu tố đầu ra và sự phân bổ yếu tố đầu ra cho yếu tố đầu vào trong sự cân bằng cấu trúc của chỉ số ROA nhằm mục đích xác định rủi ro hoạt động của ngân hàng. Bên cạnh đó, mô hình DuPont giải thích các giới hạn đo lường của các chỉ số thống kê và hồi quy. Trong ngữ cảnh nghiên cứu, phương pháp thống kê được trình bày là phương pháp hồi quy OLS. Qua đó, bằng chứng thực nghiệm dựa trên dữ liệu khảo sát bảng của 31 ngân hàng tại Việt Nam trong giai đoạn 2005-2018 và kết quả nghiên cứu cho thấy sự yếu kém trong hệ thống ngân hàng tại Việt Nam: (1) về khả năng tạo tính hấp dẫn vốn cho vay của yếu tố đầu ra và (2) sự lấn át của chi phí hoạt động ảnh hưởng đến sự đóng góp thuế thu nhập doanh nghiệp vào ngân sách nhà nước.

\section{ABSTRACT}

The purpose of this paper is not only to find out the limits of ROA. Based on the model of DuPont, factors of decomposition formula represent contributed rate of inputs for outputs and rational rate of outputs for inputs in the structural equilibrium of ROA index to determine operating risks in banking. And, DuPont model explains the limitation of measurement for statistical index and regression. In the context, the research method in the model is OLS techniques. Through the empirical evidence in the panel-data survey of 31 Vietnam banks in the periods of 2005-2018, the results have shown the weaknesses in the banking system of Vietnam: (1) the ability to attract customers' loans in output factors, (2) crowding-out 
effect of bank-operating cost on the contribution of corporate

income tax to state-budget.

\section{Giới thiệu}

Nghiên cứu yếu tố rủi ro trong lĩnh vực ngân hàng đóng vai trò quan trọng trong việc quản trị rủi ro ngân hàng, được giới học thuật và chuyên gia trong ngành quan tâm. Nhiều yếu tố rủi ro ngân hàng được các nghiên cứu trước đây xác định: năng lực điều hành và cấu trúc sở hữu liên quan đến hiệu quả hoạt động và rủi ro ngân hàng (Brickley \& James, 1987), hiệu quả hoạt động ảnh hưởng đến tỷ lệ nợ xấu ngân hàng (Jiménez, Lopez, \& Saurina, 2013; Zhang, Cai, Dickinson, \& Kutan, 2016), mức độ chấp nhận rủi ro và ổn định tài chính (Ariss, 2010; Berger, Klapper, \& Turk-Ariss, 2009; Fiordelisi \& Mare, 2014; Forssbæck \& Shehzad, 2014; Lepetit \& Strobel, 2015; Mohsni \& Otchere, 2014; Tabak, Gomes, \& Medeiros, 2015). Tuy nhiên, các nghiên cứu trước đây vẫn còn khe hở nghiên cứu về rủi ro hoạt động ngân hàng, qua đó tác giả dựa trên sự phân rã của mô hình DuPont để làm rõ rủi ro hoạt động dựa trên lý thuyết chi phí đại diện (Agency Cost Theory) về sự cân bằng hiệu quả giữa yếu tố đầu vào và đầu ra (Jensen \& Meckling, 1976). Do đó, một số vấn đề được đặt ra trong bối cảnh nghiên cứu học thuật:

Ở góc độ lý thuyết chi phí đại diện, sự cân bằng giữa yếu tố đầu ra và đầu vào của hoạt động ảnh hưởng đến hiệu quả lợi ích và chi phí, qua đó sự mâu thuẫn quyền lợi giữa người đại diện và người sở hữu cổ phần làm giảm đi hiệu quả hoạt động của ngân hàng. Vấn đề đặt ra trong bối cảnh nghiên cứu là tìm kiếm sự mất cân bằng giữa yếu tố đầu ra và đầu vào của hoạt động ngân hàng.

Về phương pháp nghiên cứu định lượng, các yếu tố đầu ra và đầu vào của mô hình DuPont trong chỉ số ROA là các chỉ số tích lũy và tương đối, do đó các yếu tố này sẽ không có ý nghĩa kinh tế và tài chính hoặc ước lượng chệnh nếu sử dụng phép toán cộng, trung bình cơ bản trong thống kê và hồi quy. Chẳng hạn, chỉ số tích lũy (tổng tài sản, vốn chủ sở hữu) là những chỉ số không thể thực hiện phép toán cộng, trung bình trong dữ liệu thời gian vì không có ý nghĩa kinh tế và tài chính; hoặc chỉ số tương đối ước lượng chệnh nếu mẫu số của chỉ số tương đối không đồng nhất. Để khắc phục nhược điểm trong phương pháp nghiên cứu định lượng, việc sử dụng mô hình DuPont và lấy cơ số logarít để giải thích rõ ý nghĩa của các yếu tố đầu ra và đầu vào trong chỉ số ROA.

Qua đó, cấu trúc của bài nghiên cứu gồm bốn phần: một là giới thiệu vấn đề nghiên cứu về lý luận rủi ro hoạt động ngân hàng và đưa ra giới hạn của chỉ số ROA trong phương pháp nghiên cứu định lượng, hai là tiếp cận cơ sở lý thuyết chi phí đại diện và đề xuất mô hình DuPont, ba là trình bày phương pháp nghiên cứu định lượng để kiểm định sự cân bằng yếu tố đầu ra và đầu vào trong cấu trúc của chỉ số $\mathrm{ROA}$, bốn là đưa ra kết quả nghiên cứu và suy luận thống kê.

\section{Cơ sở lý thuyết và mô hình nghiên cứu}

\subsection{Cơ sở lý thuyết}

Chi phí đại diện là chi phí phát sinh khi xảy ra mâu thuẫn giữa người đại diện và các cổ đông sở hữu cổ phần ngân hàng, qua đó người đại diện thay mặt cho người sở hữu điều hành hoạt động ngân hàng. Trong nghiên cứu của Jensen và Meckling (1976), sự cân bằng hiệu quả 
giữa lợi ích và chi phí trong hoạt động kinh doanh đạt trạng thái bằng không trong điều kiện bỏ qua chi phí người đại diện. Một số nghiên cứu cho thấy, chi phí đại diện tạo ra rủi ro từ hiệu quả hoạt động kinh doanh nếu chi phí hoạt động vượt ngưỡng chi phí vốn chủ sở hữu. Khi đó việc sử dụng đồng vốn trong điều hành hoạt động cao hơn chi phí sử dụng vốn thông qua cổ tức mà người sở hữu được nhận, đồng thời làm giảm khả năng tích lũy vốn chủ sở hữu (Jensen, 2005; Moh'd, Perry, \& Rimbey , 1995). Một số nghiên cứu trước đây nhận diện mức độ rủi ro trong hoạt động: nghiên cứu chi phí tài chính của các khoản nợ vay (Jensen \& Smith, 2000), năng lực điều hành và cấu trúc sở hữu liên quan đến hiệu quả hoạt động và rủi ro ngân hàng (Brickley \& James, 1987), nghiên cứu mối quan hệ giữa chi phí đại diện và tính kinh tế theo quy mô (Kochhar, 1996), nghiên cứu mối quan hệ giữa chi phí đại diện và kiểm soát báo cáo tài chính (Watts \& Zimmerman, 1983).

\subsection{Mô hình nghiên cứu}

Việc tiếp cận lý luận chi phí đại diện để xác định rủi ro từ hiệu quả hoạt động ngân hàng dựa trên hiệu quả tối ưu về sự cân bằng giữa lợi ích và chi phí theo nghiên cứu Jensen và Meckling (1976). Tuy nhiên, việc phân tích rủi ro ngân hàng trên cơ sở sự cân bằng của yếu tố đầu ra gồm tổng thu nhập lãi vay $(T R)$ và các yếu tố đầu vào gồm tổng chi phí lãi vay $(C O G S)$, tổng chi phí hoạt động $(O C)$, thuế thu nhập doanh nghiệp $(T A X)$. Khi đó, lợi nhuận ròng $(N I)$ là kết quả của hiệu quả hoạt động của ngân hàng. Theo nghiên cứu của Jensen và Meckling (1976), khi chi phí đại diện tăng thì kết quả lợi nhuận ròng $(N I)$ giảm; bên cạnh đó, việc đo lường sự đóng góp hiệu quả phân bổ tổng thu nhập lãi vay $(T R)$ một cách hợp lý sẽ nhận diện được rủi ro từ hiệu quả hoạt động, hoặc ngược lại, sự phân bổ hiệu quả vào chi phí hoạt động có tạo động lực kích thích dòng vốn cho vay của ngân hàng.

Để đánh giá sự cân bằng hiệu quả giữa các yếu tố đầu ra hoạt động, việc vận dụng công thức phân rã DuPont phản ánh yếu tố đầu ra trong chỉ số ROA (Rakicevic, Milosevic, Petrovic, \& Radojevic, 2015; Soliman, 2008):

$$
R O A=\frac{N I}{T A}=\frac{N I}{T R} \cdot \frac{T R}{T A}
$$

Trong đó chỉ số $\frac{N I}{T R}$ là chỉ số cơ cấu lợi nhuận ròng $(N I)$ trên tổng thu nhập lãi vay $(T R)$, thể hiện một đồng lãi vay mà ngân hàng kiếm được thì lợi nhuận ròng của ngân hàng chiếm tỷ lệ $\frac{N I}{T R}$ đồng; chỉ số $\frac{T R}{T A}$ là chỉ số định giá tính hấp dẫn của đồng lãi cho vay, thể hiện một đồng tài sản cho vay tạo ra $\frac{T R}{T A}$ đồng thu nhập lãi vay. Biến trung gian tổng thu nhập lãi vay $(T R)$ được xem là trạng thái không đổi của phân rã DuPont thể hiện trong công thức (1). Tuy nhiên, để thấy được sự phân bổ hiệu quả của tổng thu nhập lãi vay $(T R)$ vào các yếu tố đầu vào, sự đóng góp của hai thành phần của chỉ số $\frac{N I}{T R}$ và chỉ số $\frac{T R}{T A}$ là khác nhau.

Bên cạnh đó, việc đánh giá sự đóng góp của yếu tố đầu vào ảnh hưởng đến yếu tố đầu ra, công thức phân rã DuPont phản ánh yếu tố đầu vào trong chỉ số $R O A$ :

$$
R O A=\frac{N I}{T A}=\frac{N I}{E B T} \cdot \frac{E B T}{G P R} \cdot \frac{G P R}{C O G S} \cdot \frac{C O G S}{T A}
$$

Trong đó chỉ số $\frac{N I}{E B T}$ là chỉ số cơ cấu lợi nhuận ròng $(N I)$ trên lợi nhuận trước thuế $(E B T)$, thể hiện gánh nặng thuế thu nhập doanh nghiệp của ngân hàng khi chỉ số này tiệm cận về 0 ; chỉ số $\frac{E B T}{G P R}$ là chỉ số cơ cấu lợi nhuận trước thuế ( $\left.E B T\right)$ trên lợi nhuận gộp $(G P R)$, thể hiện 
gánh nặng chi phí hoạt động của ngân hàng khi chỉ số này tiệm cận về 0 ; chỉ số $\frac{G P R}{C O G S}$ là chỉ số cơ cấu lợi nhuận gộp $(G P R)$ trên tổng chi phí lãi vay $(C O G S)$, thể hiện biên lợi nhuận của ngân hàng trên giá vốn, thể hiện đồng lời từ hoạt động tín dụng; chỉ số $\frac{C O G S}{T A}$ là chỉ số định giá tổng chi phí lãi vay $(C O G S)$ trên tổng tài sản $(T A)$, thể hiện một đồng tài sản cho khách hàng vay thì ngân hàng phải tốn chi phí lãi vay $\frac{C O G S}{T A}$ đồng. Dựa trên sự cân bằng đầu ra và đầu vào của hoạt động kinh doanh của ngân hàng:

$$
R O A=\frac{N I}{T R} \cdot \frac{T R}{T A}=\frac{N I}{E B T} \cdot \frac{E B T}{G P R} \cdot \frac{G P R}{C O G S} \cdot \frac{C O G S}{T A}
$$

Lấy cơ số logarit hai vế từ công thức phân rã DuPont của chỉ số $R O A$, ý nghĩa của sự cân bằng đầu vào và đầu vào trong tài chính xem xét mức độ đóng góp theo hai khía cạnh:

(1) Mức độ đóng góp của yếu tố đầu vào để tạo ra giá trị thặng dư cho yếu tố đầu ra của hoạt động ngân hàng thông qua hệ phương trình đồng thời:

$$
\begin{aligned}
& \ln \frac{N I}{T R}=\beta_{0}+\beta_{1} \ln \frac{N I}{E B T}+\beta_{2} \ln \frac{E B T}{G P R}+\beta_{3} \ln \frac{G P R}{C O G S}+\beta_{4} \ln \frac{C O G S}{T A}+\varepsilon_{1} \\
& \ln \frac{T R}{T A}=\alpha_{0}+\alpha_{1} \ln \frac{N I}{E B T}+\alpha_{2} \ln \frac{E B T}{G P R}+\alpha_{3} \ln \frac{G P R}{C O G S}+\alpha_{4} \ln \frac{C O G S}{T A}+\varepsilon_{2}
\end{aligned}
$$

Trong đó sự cân bằng của các tham số ước lượng về mức độ đóng góp của yếu tố đầu vào để tạo ra giá trị thặng dư cho yếu tố đầu ra thể hiện ở phương trình (4a) và $(4 \mathrm{~b}) \beta_{0}+\alpha_{0}=$ 0 và $\beta_{i}+\alpha_{i}=1$ với $i=\{1 \div 4\}$

(2) Mức độ phân bổ của yếu tố đầu ra cho yếu tố đầu ra của hoạt động ngân hàng thông qua hệ phương trình đồng thời

$$
\begin{aligned}
& \ln \frac{N I}{E B T}=\gamma_{0}+\gamma_{1} \ln \frac{N I}{T R}+\gamma_{2} \ln \frac{T R}{T A}+\varepsilon_{3} \\
& \ln \frac{E B T}{G P R}=\pi_{0}+\pi_{1} \ln \frac{N I}{T R}+\pi_{2} \ln \frac{T R}{T A}+\varepsilon_{4} \\
& \ln \frac{G P R}{C O G S}=\tau_{0}+\tau_{1} \ln \frac{N I}{T R}+\tau_{2} \ln \frac{T R}{T A}+\varepsilon_{5} \\
& \ln \frac{C O G S}{T A}=\omega_{0}+\omega_{1} \ln \frac{N I}{T R}+\omega_{2} \ln \frac{T R}{T A}+\omega_{6}
\end{aligned}
$$

Trong đó sự cân bằng của các tham số ước lượng trong việc phân bổ của yếu tố đầu ra cho yếu tố đầu vào tại phương trình $(5 \mathrm{a}),(5 \mathrm{~b}),(5 \mathrm{c}),(5 \mathrm{~d}) \gamma_{0}+\pi_{0}+\tau_{0}+\omega_{0}=0$ và $\gamma_{j}+\pi_{j}+$ $\tau_{j}+\omega_{j}=1$ với $j=[1 \div 2\}$

Cấu trúc cân bằng dựa vào sự phân rã DuPont trong chỉ số $R O A$ giữa yếu tố đầu vào và yếu tố đầu ra trong hoạt động kinh doanh của ngân hàng, trong đó hai phương trình $(4 \mathrm{a}, 4 \mathrm{~b})$ đánh giá mức độ đóng góp của yếu tố đầu vào đến yếu tố đầu ra; và bốn phương trình $(5 \mathrm{a}, 5 \mathrm{~b}$, $5 \mathrm{c}, 5 \mathrm{~d})$ đánh giá mức độ phân bổ của yếu tố đầu ra đến yếu tố đầu vào.

\section{Phương pháp và dữ liệu nghiên cứu}

\subsection{Kiểm định giả thuyết}

Công thức phân rã Dupont của chỉ số $R O A$ là cách tiếp cận mới về phương pháp luận dựa trên sự cân bằng cấu trúc giữa các yếu tố đầu vào và các yếu tố đầu ra trong phân tích hồi quy, trong đó công cụ được sử dụng mô hình là kỹ thuật OLS để tính tham số ước lượng và sử 
dụng kiểm định trung bình $\mathrm{T}$-Test để kiểm tra mức độ đóng góp của các tham số:

- Nếu mức ý nghĩa thống kê sig.t(tầu vào i) $>.05$, cho thấy rằng yếu tố đầu vào thứ $i$ không thấy sự đóng góp cho yếu tố đầu ra thứ j.

- Nếu mức ý nghĩa thống kê sig.t(đầu ra j) $>.05$, cho thấy rằng yếu tố đầu ra thứ j không thấy sự phân bổ cho yểu tố đầu vào thứ i.

\subsection{Dũ liệu nghiên cúu}

Cơ sở dữ liệu là dữ liệu bảng không cân bằng của 31 ngân hàng tại Việt Nam trong giai đoạn 2005-2018 được trích từ nguồn Bankscope và Orbis Bank Focus, các biến quan sát ban đầu trong mô hình nghiên cứu gồm lợi nhuận ròng $(\mathrm{NI})$, lợi nhuận trước thuế (EBT), lợi nhuận gộp (GPR), tổng chi phí lãi vay (COGS), tổng thu nhập lãi vay (TR), và tổng tài sản (TA) và toàn bộ các biến quan sát ban đầu có đơn vị tính là triệu đồng/năm. Qua đó, các yếu tố đầu vào và đầu ra của chỉ số ROA trong mô hình nghiên cứu được thiết lập như sau:

\section{Bảng 1}

Định nghĩa các biến đầu vào và đầu ra trong mô hình nghiên cứu

\begin{tabular}{|c|c|c|}
\hline Tên biến & Định nghĩa & Giải thích ý nghĩa tài chính \\
\hline \multicolumn{3}{|c|}{ Yếu tố đầu ra của hoạt động ngân hàng } \\
\hline $\ln \frac{N I}{T R}$ & $\begin{array}{l}\text { Logarit của tỷ lệ lợi nhuận ròng trên } \\
\text { tồng thu nhập lãi vay }\end{array}$ & $\begin{array}{l}\text { Trong một đồng thu nhập lãi vay, } \\
\text { lợi nhuận ròng chiếm NI/TR } \\
\text { dồng }\end{array}$ \\
\hline $\ln \frac{T R}{T A}$ & $\begin{array}{l}\text { Logarit của tỷ lệ tổng thu nhập lãi } \\
\text { vay trên tổng tài sản }\end{array}$ & $\begin{array}{l}\text { Trong một đồng tài sản, thu nhập } \\
\text { lãi vay kiếm được TR/TA đồng }\end{array}$ \\
\hline \multicolumn{3}{|c|}{ Yếu tố đầu vào của hoạt động ngân hàng } \\
\hline $\ln \frac{N I}{E B T}$ & $\begin{array}{l}\text { Logarit của tỷ lệ lợi nhuận ròng trên } \\
\text { lợi nhuận trước thuế }\end{array}$ & $\begin{array}{l}\text { Gánh nặng thuế thu nhập doanh } \\
\text { nghiệp }\end{array}$ \\
\hline $\ln \frac{E B T}{G P R}$ & $\begin{array}{l}\text { Logarit của tỷ lệ lợi nhuận trước } \\
\text { thuế trên lợi nhuận gộp }\end{array}$ & $\begin{array}{l}\text { Gánh nặng chi phí hoạt động của } \\
\text { ngân hàng }\end{array}$ \\
\hline $\ln \frac{G P R}{C O G S}$ & $\begin{array}{l}\text { Logarit của tỷ lệ lợi nhuận gộp trên } \\
\text { tồng chi phí lãi vay }\end{array}$ & $\begin{array}{l}\text { Trong một đồng chi phi lãi vay, } \\
\text { lợi nhuận của ngân hàng kiếm } \\
\text { được GPR/COGS dồng }\end{array}$ \\
\hline $\ln \frac{C O G S}{T A}$ & $\begin{array}{l}\text { Logarit của tỷ lệ tổng chi phí lãi vay } \\
\text { trên tổng tài sản }\end{array}$ & $\begin{array}{l}\text { Trong một đồng tài sản, chi phí } \\
\text { lãi vay tôn COGS/TA đồng }\end{array}$ \\
\hline
\end{tabular}

Ghi chú: Có 03 quan sát có tỷ lệ âm được loại khỏi dữ liệu nghiên cứu trước khi lấy cơ số logarit.

Nguồn: Kết quả phân tích dữ liệu của nhóm nghiên cứu

\subsection{Mô tả thống kê các biến ngành ngân hàng}

Sự cân bằng cấu trúc dựa trên sự phân rã cấu trúc DuPont của chỉ số ROA được vận dụng trong hồi quy; tuy nhiên, các biến số trong mô hình nghiên cứu là các biến số tỷ lệ do đó để tính được tỷ lệ bình quân của ngành ngân hàng cần được vận dụng tính tổng của các ngân hàng theo dữ liệu chéo.

Qua kết quả biến số tích lũy cho thấy rằng số liệu thống kê của biến quan sát tổng tài sản (TA) không thể tính vì không ý nghĩa kinh tế vì biến số tổng tài sản (TA) là biến số tích lũy trong bảng cân đối kế toán. 
Qua kết quả bảng 2, tỷ lệ bình quân của các yếu tố đầu vào và đầu ra của hoạt động kinh doanh ngành ngân hàng trong mô trong giai đoạn 2005-2018 của 31 ngân hàng: tỷ lệ lợi nhuận ròng trên tổng thu nhập lãi vay của ngành đạt $\frac{\sum N I_{k}}{\sum T R_{k}}=0.1086$; tỷ lệ lợi nhuận ròng trên lợi nhuận trước thuế của ngành đạt $\frac{\sum N I_{k}}{\sum E B T_{k}}=0.7792$; tỷ lệ lợi nhuận trước thuế trên lợi nhuận gộp của ngành đạt $\frac{\sum E B T_{k}}{\sum G P R_{k}}=0.3758$; tỷ lệ lợi nhuận gộp trên tổng chi phí lãi vay của ngành đạt $\frac{\sum G P R_{k}}{\sum C O G S_{k}}=$ 0.5890 .

\section{Bảng 2}

Kết quả các thống kê tỷ lệ bình quân ngành (phân loại theo năm)

\begin{tabular}{|c|c|c|c|c|c|c|c|c|c|}
\hline STT & Năm & $\begin{array}{c}\text { Số } \\
\text { ngân } \\
\text { hàng }\end{array}$ & ROA & NI/TR & TR/TA & NI/EBT & EBT/GPR & GPR/COGS & COGS/TA \\
\hline 1 & 2005 & 18 & 0.0077 & 0.1121 & 0.0688 & 0.7477 & 0.3447 & 0.7698 & 0.0389 \\
\hline 2 & 2006 & 25 & 0.0091 & 0.1266 & 0.0722 & 0.7572 & 0.4483 & 0.5951 & 0.0453 \\
\hline 3 & 2007 & 26 & 0.0120 & 0.1716 & 0.0696 & 0.7941 & 0.5973 & 0.5672 & 0.0444 \\
\hline 4 & 2008 & 28 & 0.0109 & 0.1075 & 0.1013 & 0.7941 & 0.4570 & 0.4207 & 0.0713 \\
\hline 5 & 2009 & 29 & 0.0126 & 0.1866 & 0.0677 & 0.7769 & 0.6585 & 0.5739 & 0.0430 \\
\hline 6 & 2010 & 30 & 0.0104 & 0.1286 & 0.0806 & 0.7683 & 0.5147 & 0.4818 & 0.0544 \\
\hline 7 & 2011 & 30 & 0.0101 & 0.0895 & 0.1132 & 0.7456 & 0.4049 & 0.4214 & 0.0796 \\
\hline 8 & 2012 & 29 & 0.0078 & 0.0761 & 0.1028 & 0.7524 & 0.3161 & 0.4704 & 0.0699 \\
\hline 9 & 2013 & 30 & 0.0062 & 0.0800 & 0.0775 & 0.7557 & 0.3128 & 0.5116 & 0.0512 \\
\hline 10 & 2014 & 27 & 0.0061 & 0.0921 & 0.0657 & 0.7838 & 0.3103 & 0.6097 & 0.0408 \\
\hline 11 & 2015 & 26 & 0.0058 & 0.0951 & 0.0607 & 0.7794 & 0.2850 & 0.7485 & 0.0347 \\
\hline 12 & 2016 & 24 & 0.0062 & 0.1000 & 0.0623 & 0.7962 & 0.2990 & 0.7236 & 0.0361 \\
\hline 13 & 2017 & 24 & 0.0074 & 0.1152 & 0.0640 & 0.7970 & 0.3441 & 0.7247 & 0.0371 \\
\hline 14 & 2018 & 22 & 0.0099 & 0.1466 & 0.0676 & 0.7986 & 0.4447 & 0.7029 & 0.0397 \\
\hline \multicolumn{3}{|c|}{ Bình quân } & & 0.1086 & & 0.7792 & 0.3758 & 0.5890 & \\
\hline
\end{tabular}

Nguồn: Số liệu tính toán của tác giả

\section{Kết quả nghiên cứu}

\subsection{Kết quả nghiên cúu}

Sự phân rã DuPont của chỉ số ROA với 06 phương trình cân bằng cấu trúc giữa các yếu tố đầu vào và đầu ra của hoạt động kinh doanh trong lĩnh vực ngân hàng trong giai đoạn 20052018 như sau:

Kết quả hồi quy phương trình (5a) trong bảng 3 cho thấy rằng mức ý nghĩa thống kê của biến $\ln \frac{N I}{T R}$ và $\ln \frac{T R}{T A}$ lớn hơn 0.05 cho thấy rằng các yếu tố đầu ra của chỉ số $\mathrm{ROA}$ không có sự phân bổ đối với yếu tố tỷ lệ lợi nhuận ròng trên lợi nhuận trước thuế $\ln \frac{N I}{E B T}$ trong lĩnh vực ngành ngân hàng với mức ý nghĩa kiểm định phương sai sig. $\left(\mathrm{R}^{2}\right)=.075>.05$. 


\section{Bảng 3}

Phân tích hồi quy dựa trên sự phân rã DuPont của chỉ số ROA

\begin{tabular}{|c|c|c|c|c|c|c|}
\hline Tên biến & $\begin{array}{l}(4 a) \\
\frac{N I}{T R}\end{array}$ & $\begin{array}{l}(4 \mathrm{~b}) \\
\frac{T R}{T A}\end{array}$ & $\begin{array}{l}(5 \mathrm{a}) \\
N I \\
E B T\end{array}$ & $\begin{array}{c}(5 b) \\
E B T \\
G P R\end{array}$ & $\begin{array}{c}(5 \mathrm{c}) \\
\mathrm{GPR} \\
C O G S\end{array}$ & $\frac{\begin{array}{c}(5 \mathrm{~d}) \\
C O G S\end{array}}{T A}$ \\
\hline Hằng số & $\begin{array}{r}-.5263 * * * \\
(.0396)\end{array}$ & $\begin{array}{r}.5263 * * * \\
(.0396)\end{array}$ & $\begin{array}{r}-.2784 * * * \\
(.0388)\end{array}$ & $\begin{array}{r}1.7165 * * * \\
(.1777)\end{array}$ & $\begin{array}{r}-1.404 * * * \\
(.2497)\end{array}$ & $\begin{array}{r}-.2977 * * \\
(.0802)\end{array}$ \\
\hline$\frac{N I}{T R}$ & & & $\begin{array}{l}.0076^{*} \\
(.0042) \\
\end{array}$ & $\begin{array}{r}.8216^{* * *} \\
(.0191) \\
\end{array}$ & $\begin{array}{r}.2514 * * * \\
(.0268) \\
\end{array}$ & $\begin{array}{r}-.0805 * * * \\
(.0086) \\
\end{array}$ \\
\hline$\frac{T R}{T A}$ & & & $\begin{array}{r}-.0138 \\
(.0136) \\
\end{array}$ & $\begin{array}{r}.2920 * * * \\
(.0623) \\
\end{array}$ & $\begin{array}{r}-.4072 * * * \\
(.0876) \\
\end{array}$ & $\begin{array}{r}1.1290 * * * \\
(.0281) \\
\end{array}$ \\
\hline$\frac{N I}{E B T}$ & $\begin{array}{r}.8607 * * * \\
(.0413)\end{array}$ & $\begin{array}{r}.1393 * * * \\
(.0413)\end{array}$ & & & & \\
\hline$\frac{E B T}{G P R}$ & $\begin{array}{r}.9914 * * * \\
(.0037)\end{array}$ & $\begin{array}{r}.0086^{* *} \\
(.0037)\end{array}$ & & & & \\
\hline$\frac{G P R}{C O G S}$ & $\begin{array}{r}.7217 * * * \\
(.0074)\end{array}$ & $\begin{array}{r}.2783 * * * \\
(.0074)\end{array}$ & & & & \\
\hline$\frac{C O G S}{T A}$ & $\begin{array}{r}.0491 * * * \\
(.0107)\end{array}$ & $\begin{array}{r}.9509 * * * \\
(.0107)\end{array}$ & & & & \\
\hline Số quan sát & 368 & 368 & 368 & 368 & 368 & 368 \\
\hline Hệ số $\mathrm{R}^{2}$ & $.9962 * * *$ & $.9600 * * *$ & .0141 & $.8365 * * *$ & $.2643 * * *$ & $.8399 * * *$ \\
\hline
\end{tabular}

Ghi chú: *** nhỏ hơn mức ý nghĩa thống kê 0.01 ;** nhỏ hơn 0.05 ; * nhỏ hơn 0.1 Nguồn: Kết quả xử lý từ dữ liệu điều tra

\subsection{Suy luận}

Cân bằng cấu trúc của chỉ số ROA cho thấy rằng, có những nút thắt cần được tháo gỡ trong hoạt động của ngành ngân hàng, chẳng hạn như mối tương quan giữa gánh nặng chi phí hoạt động chưa tạo được động lực và tính hấp dẫn tài sản cho vay của ngân hàng, đồng thời mối tương quan giữa yếu tố đầu ra của chỉ số ROA chưa phản ánh được mức độ phân bổ cho thuế thu nhập doanh nghiệp. Trong điều kiện các ngân hàng hoạt động độc lập, tỷ lệ ROA của ngành đạt mức bình quân $0.00662 \sim 0.662 \%$, trong đó:

Tỷ lệ đóng góp của yếu tố đầu vào cho yếu tố đầu ra trong mô hình ước lượng

$$
R O A=\frac{N I}{T R} \cdot \frac{T R}{T A}=.1508 \cdot .0439=.00662
$$

Kết quả ước lượng cho thấy rằng, hiệu suất của yếu tố tổng thu nhập lãi vay trên tổng tài sản của ngành ngân hàng trong giai đoạn 2005-2018 chưa tạo tính hấp dẫn đối với yếu tố đầu ra của ngành ngân hàng vì tổng thu nhập lãi vay trên tổng tài sản chỉ đạt .0439 thấp hơn tổng chi phí lãi vay trên tổng tài sản .0645 .

Tỷ lệ phân bổ của yếu tố đầu ra cho yếu tố đầu vào trong mô hình ước lượng 


$$
R O A=\frac{N I}{E B T} \cdot \frac{E B T}{G P R} \cdot \frac{G P R}{C O G S} \cdot \frac{C O G S}{T A}=1.0178 \cdot .0642 \cdot 1.5695 \cdot .0645=.00662
$$

Kết quả ước lượng cũng chỉ ra tỷ lệ nhuận gộp trên tổng chi phí lãi vay của ngành đạt 1.5695 trong khi đó hiệu suất đóng góp của thuế thu nhập doanh nghiệp cho ngân sách nhà nước chưa đạt kỳ vọng (vì $1.0178>1$ ) do bị chi phí hoạt động lấn át với tỷ lệ lợi nhuận trước thuế trên lợi nhuận gộp chỉ đạt .0642 trong ngành ngân hàng.

\section{Kết luận}

Sự phân rã DuPont của chỉ số ROA trong hồi quy là cách tiếp cận dựa trên sự cân bằng cấu trúc giữa các yếu tố đầu vào và yếu tố đầu ra trong hệ thống báo cáo tài chính của ngành ngân hàng, qua đó sự phân rã cấu trúc ROA khai thác lợi thế so sánh, điểm mạnh và điểm yếu để chỉ ra rủi ro hoạt động của ngành ngân hàng. Kết quả nghiên cứu dựa trên số liệu báo cáo tài chính của 31 ngân hàng trong giai đoạn 2005-2018 cho thấy có ba thành phần cần khắc phục: khả năng tạo tính hấp dẫn vốn cho vay của yếu tố đầu ra; sự lấn át chi phi hoạt động ảnh hưởng đến sự đóng góp thuế thu nhập doanh nghiệp trong ngành ngân hàng.

\section{Tài liệu tham khảo}

Ariss, R. T. (2010). On the implications of market power in banking: Evidence from developing countries. Journal of Banking and Finance, 34(4), 765-775. doi:10.1016/j.jbankfin.2009.09.004

Berger, A. N., Klapper, L. F., \& Turk-Ariss, R. (2009). Bank competition and financial stability. Journal of Financial Services Research, 35(2) 99-118. doi:10.1007/s10693-009-0065-8

Brickley, J. A., \& James, C. M. (1987). The takeover market, corporate board composition, and ownership structure: The case of banking. Journal of Law and Economics, 30, 161-180. doi:10.1086/467134

Fiordelisi, F., \& Mare, D. (2014). Competition and financial stability in European cooperative banks. Journal of International Money and Finance, 45, 1-16. doi:10.1016/j.jimonfin.2014.02.008

Forssbæck, J., \& Shehzad, C. T. (2014). The conditional effects of market power on bank risk: Cross-country evidence. Review of Finance, 1-40. doi:10.1093/rof/rfu044

Jensen, M. C. (2005). Agency costs of overvalued equity. Financial Management, 34(1), 5-19. doi:10.1111/j.1755-053x.2005.tb00090.x

Jensen, M. C., \& Meckling, W. H. (1976). Theory of the firm: Managerial behavior, agency costs and ownership structure. Journal of Financial Economics, 3(4), 305-360. doi:10.1016/0304-405X(76)90026-X

Jensen, M. C., \& Smith, C. W., Jr. (2000). Stockholder, manager, and creditor interests: Applications of agency theory. SSRN Electronic Journal. doi:10.2139/ssrn.173461

Jiménez, G., Lopez, J. A., \& Saurina, J. (2013). How does competition affect bank risk-taking? Journal of Financial Stability, 9, 185-195. doi:10.1016/j.jfs.2013.02.004 
Kochhar, R. (1996). Explaining firm capital structure: The role of agency theory vs. transaction cost economics. Strategic Management Journal, 17(9), 713-728. doi:10.1002/(SICI)1097-0266(199611)17:9<713::AID-SMJ844>3.0.CO;2-9

Lepetit, L., \& Strobel, F. (2015). Bank insolvency risk and Z-Score measures: A refinement. Finance Research Letter, 13, 214-224. doi:10.1016/j.frl.2015.01.001

Mohsni, S., \& Otchere, I. (2014). Financial crisis, liquidity infusion and risk-taking: The case of Canadian banks. Journal of Banking Regulation, 16(2), 146-167. doi:10.1057/jbr.2014.2

Moh'd, M. A., Perry, L. G., \& Rimbey, J. N. (1995). An investigation of dynamic relationship between agency theory and dividend policy. The Financial Review, 30, 367-385.

Rakicevic, A., Milosevic, P., Petrovic, B., \& Radojevic, D. G. (2015). DuPont financial ratio analysis using logical aggregation. Advances in Intelligent Systems and Computing, 727739. doi:10.1007/978-3-319-18416-6_57

Soliman, M. T. (2008). The use of DuPont analysis by market participants. Accounting Review, 83(3), 823-853. doi:10.2308/accr.2008.83.3.823

Tabak, B. M., Gomes, G. M. R., \& Medeiros, M. (2015). The impact of market power at bank level in risk-taking: The Brazilian case. International Review of Financial Analysis, 40, 154-165. doi:10.1016/j.irfa.2015.05.014

Watts, R. L., \& Zimmerman, J. L. (1983). Agency problems, auditing, and the theory of the firm: Some evidence. Journal of Law and Economics, 26(3), 613-633. doi:10.1086/467051

Zhang, D., Cai, J., Dickinson, D. G., \& Kutan, A. M. (2016). Non-performing loans, moral hazard and regulation of the Chinese commercial banking system. Journal of Banking and Finance, 63, 48-60. doi:10.1016/j.jbankfin.2015.11.010 